Aus der Inneren Abteilung des Allgemeinen Krankenhauses in Lübeck. (Oberarzt: Dr. Hofstaetter.)

\title{
Akute gelbe Leberatrophie bei sekundärer Lues.
}

Von Dr. Wilhelm Graef.

Wie die akute gelbe Leberatrophie überhaupt eine recht seltene Erkrankung darstellt, so sind im besonderen Fälle auf luetischer Basis nur in geringer Anzahl bekannt geworden. Anscheinend nimmt die Krankheit dabei ihren Ausgang von einem Ikterus, wie er im Sekundärstadium der Syphilis nicht allzu selten beobachtet wird.

Ich möchte nun im folgenden einen Fall beschreiben, der im zweiten, atrophischen Stadium in unsere Behandlung kam.

Es handelt sich um ein 22 jähriges Dienstmädchen, E. K., das im August 1908 in unserer Anstalt als II.-para einen spontanen Partus durchmachte. Mutter und Kind wurden damals als völlig gesund befunden. Nach Angabe der Mutter der Patientin soll seit 14 Tagen Gelbsucht bestehen, seit zwei Tagen Verwirrtheit und Erbrechen. Phosphorvergiftung ist auszuschließen.

Von der Patientin selbst, die am 6. April 1909 gegen Abend in das Krankenhaus aufgenommen wurde, ist eine Anamnese nicht zu erheben, da sie nicht ganz orientiert ist, zusammenhanglos spricht und mäBige motorische Unruhe zeigt. Dabei macht sich eine Sprachstörung bemerkbar, derart, daß die einzelnen Worte verkehrt ausgesprochen und dann mehrmals wiederholt werden. Es besteht ziemlich häufiges, nicht blutiges Erbrechen. Kein Fieber. Puls gut. Leib weich, nicht druckempfindlich. Die Leberdämpfung reicht in der Mamillarlinie nur bis etwa 11/2 Querfinger oberhalb des Rippenbogens. Die genauere Untersuchung am 7. April ergibt folgendes: mäßiger $\mathbf{E r}$ nährungszustand, graziler Körperbau, starke ikterische Verfärbung der Haut und der Skleren. An Rumpf und Extremitäten pustulöser Ausschlag. Am linken Ober- und Unterschenkel fünf große Effloreszenzen von $1 / 2-1 \mathrm{~cm}$ Durchmesser mit scharfen Grenzen und von rotbrauner Farbe. Inguinaldrüsen beiderseits stark rosenkranzartig geschwollen. Geringes Oedem der kleinen Labien und an der Clitoris. An den kleinen Labien und an den gegenüberliegenden Stellen der großen Labien finden sich mehrere runde bis ovale, scharf abgegrenzte, flache, nässende und leicht blutende Geschwüre. An der Portio ebenfalls ein nässendes, scharfrandiges Ulcus. Uterus nicht vergrößert. Am Anus einige Rhagaden mit infiltrierten Rändern.

Der Sitz des Primäraffektes war nicht mehr. nachweisbar. Herzdämpfung normal, Töne rein. Lungen zeigen überall hellen Schall und reines vesikuläres Atmen. Abdomen etwas eingezogen, nirgends druckempfindlich, auch nicht in der Lebergegend. Leber nicht palpabel, untere Dämpfungsgrenze steht in der Mamillarlinie drei Querfinger über dem Rippenbogen. Milz. nicht palpabel, perkutorisch keine Vergrößerung nachweisbar. Patellarreflexe vorhanden, Pupillen mittelweit, trăge reagierend. Urin wird in reichlicher Menge spontan gelassen. Bierbraune Farbe, Reaktion sauer, spezifisches Gewicht 1016, Spur EiweiB, kein Zucker, Gmelinsche und Chloroformprobe stark positiv. Im Zentrifugat vereinzelte gelb gefärbte, granulierte Zylinder, Epithelialzylinder, Epithelien der verschiedensten Formen, Epitheldetritus, weiße und ausgelaugte rote Blutkörperchen in ziemlicher Anzahl. Im Alkoholextrakt des auf $1 / 10$ Vol. eingedampften Urins fanden sich doppelt konturierte, kugelige Gebilde und zierliche Nadelbüschel, die wir als Leuzin und Tyrosin ansprachen. Die erwähnten Gebilde waren auch im weiteren Verlauf stets im Urin vorhanden. Die von uns gesehenen Kristalle wurden von Herrn Dr. phil. Th. Wetzke (Lübeck) chemisch einwandfrei als Leuzin und Tyrosin identifiziert und waren zuletzt sehr zahlreich vorhanden. Das spezifische Gewicht des Harns schwankte zwischen 1016 und 1019. Eiweißgehalt des geklärten Urins betrug nicht mehr als $1 / 1-1 / 2$ pro mille. Urinmenge nicht vermindert, zwei Tage ante mortem noch $1350 \mathrm{ccm}$ in 24 Stunden; zuletzt ließ Patientin unter sich gehen. 
Am dritten Beobachtungstag ist in der Mamillarlinie an der fünften Rippe ein etwa fingerbreiter Bezirk etwas gedämpft mit tympanitischem Beiklang. Oberhalb voller Lungenschall, unterhalb Tympanie. Vom nächsten Tag an Leberdämpfung völlig vesrchwunden. Die Temperatur schwankte in der Beobachtungszeit zwischen 36,4 und 37,4 (Aftermessung), Puls 68-88, kräftig und regelmäßig, in den allerletzten Tagen 100-120, klein, regelmäßig. Die Herztöne bekommen zuletzt einen dumpfen, paukenden Charakter. Respirationsfrequenz wechselnd, nie erheblich beschleunigt oder verlangsamt. CheyneStokesches Atmen nicht beobachtet. Thoraxexkursionen in den letzten Tagen sehr flach. Linke Seitenlage auffallend bevorzugt. Erbrechen im Anfang stark, später mäßig, hörte einen Tag a.m. ganz auf. Blut war in dem Erbrochenen niemals nachweisbar, ebensowenig im Stuhlgang, der vollständig acholisch war. Auch die Benzidinprobe des Urins war negativ. Blutungen im Augenhintergrund (Litten) wurden nicht beobachtet. Die Nahrungsaufnahme war wegen des Erbrechens und zuletzt wegen Somnolenz gering.

Was die zerebralen Symptome anlangt, so ist der Zustand bei der A afnahme bereits geschildert. Am nächsten Tag etwas klarer, Patientin gibt bessere Antworten, dann wieder apathisch und somnolent. Dazwischen redet sie laut vor sich hin, sagt, ihr fehle garnichts. In der Nacht vom 9. auf 10. April zum ersten Male stärkere motorische Unruhe, lautes Reden und Schreien. Tagsüber wieder ruhiger, gegen Abend hochgradige Unruhe, will aus dem Bett springen, stößt dabei laute, gellende Rufe aus. Am nächsten Tag kurze Zeit wieder etwas unruhiger, verfällt sodann in Somnolenz. Am 13. April früh erfolgt Exitus im Koma.

Die serologische Prüfung des Blutes der Patientin auf Lues, welche im Hygienischen Institute zu Kiel ausgefuhrt wurde, ergab ein zweifelhaftes Resultat. Wie mir Herr Dr. Bitter liebenswürdigerweise mitteilte, bewirkte das Serum nur eine unvollständige Hemmung dergestalt, daß ein kleiner Teil der Hammelblutkörper gelöst wurde. Die Reaktion hatte zweimal den gleichen Erfolg, und dem Untersucher scheint es möglich zu sein, daß der im Blute deutlich vorhandene Gallenfarbstoff diese Störung verursacht hat.

Die am 14. April ausgeführte Sektion ergab folgendes: Die Sektion der Schädelhöhle bot keine Besonderheiten. Bei der Eröffnung der Bauchhöhle liegen die Darmschlingen frei vor. Netz nach oben geschlagen. Zwerchfellstand links oberer Rand der vierten Rippe, rechts an der vierten Rippe. Unter dem rechten Rippenbogen liegen Dünndurmschlingen, von der Leber ist auch bei kräftigem Anheben des Rippenrandes nichts zu sehen. Im Herzbeutel eine ziemlich reichliche Menge ikterisch gefärbter Flüssigkeit. Pleura- und Bauchhöhle fast frei von Flüssigkeit. Das entsprechend große Herz zeigt zahlreiche subepikardiale Blutungen, sbeonders am Sulcus coronarius. Klappen nicht verändert. Muskulatur des Herzens mürbe, gelbbräunlich, fettig glänzend. Intima aller Gefäße gelblich verfärbt. Lungen ohne wesentlichen Befund. Milz vergrößert 15:11 cm, auf Durchschnitt blaurot, ziemlich weich. Die Därme zeigen zahl reiche Sugillationen unter die Serosa, am stärksten ist das Coecum betroffen. Auch Mesenterium und Netz weisen erhebliche Blutaustritte auf. Schleimhaut des Darmes besonders in den oberen Abschnitten katarrhalisch geschwellt und injiziert. Der Magen enthält dunkle, blutige Flüssigkeit, die oberen Darmabschnitte führen blutig gefärbte Ingesta, im Rectum heller, acholischer, fester Stuhl. Mesenterialdrüsen und Drüsen an der Porta hepatis geschwollen. Schleimhaut der Harnblase stark injiziert. Die Oberfläche der Niere zeigt nach Abziehung der Kapsel vereinzelte kleine Blutungen. Durchschnitt zeigt blaß gelblichrote Farbe, fettigen Glanz, deutliche Zeichnung. Die Leber liegt in sich zusammengerollt und nach hinten gesunken auf der Wirbelsäule. Gesamtbreite $22 \mathrm{~cm}$, rechter Lappen $13 \mathrm{~cm}$ hoch, linker Lappen $10 \mathrm{~cm}$ hoch. Sie fühlt sich ganz weich und brüchig an. Unter der Glissonschen Kapsel, die verdickt, blaß und eigentümlich gerunzelt erscheint, sieht man Herde von ockergelber Farbe durchscheinen. Leberlappen abgeplattet, Ränder scharf. Der linke Lappen ist anscheinend stärker atrophisch als der rechte und mehr gleichmäßig dunkelrotgelb, während rechts ockergelbe mit dunkelrotgelben Partien abwechseln. Die roten Partien erscheinen gegenüber den gelben auf dem Durchschnitte eingesunken.

Ueber den histologischen Befund teilte mir Herr Dr. Zimmermann aus dem Pathologischen Institute zu Rostock, dem die Organe zur näheren Untersuchung ïbersandt wurden, freundlicherweise folgendes mit: In der Leber fanden sich neben Herden von hochgradig nekrotisch veränderten und verfetteten Leberzellen restierende hyperplastische Inseln von Leberzellenbalken, ferner kleinzellige Infiltrate im interlobulären Bindegewebe mit Vermehrung des letzteren (beginnende zirrhotische Veränderungen) und eine enorme Gallengangswucherung. In der. Niere fanden sich ebenfalls neben gut erhaltenen Nierenepithelien stark parenchymatös veränderte (körniger Zerfall des Protoplasmas, Fettinfiltration, Kerndegenerationen), starke Epitheldesquamation. Am Herzen
Fragmentatio cordis und hochgradige fettige Degeneration der Herzmuskelfibrillen. In der Milz im wesentlichen die Zeichen der Hyperämie. In dem Ulcus an der Portio ließen sich nur die histologischen Zeichen der Entzündung nachweisen, hyaline Veränderungen der Gefäßwände nicht.

Der im vorstehenden beschriebene Fall bietet gegenüber den sonst beobachteten keine wesentlichen Besonderheiten. Sehr selten ist allerdings der protrahierte Verlauf des zweiten, charakteristischen Stadiums der Krankheit, das hier etwa 9 Tage betrug, während es in der Regel nur 11/2-3 Tage dauert. Dementsprechend sind auch die früh einsetzenden Regenerationsprozesse, die sich schon makroskopisch durch eine größere Verbreitung der roten Partien kundgeben, gut ausgeprägt. Die Hauptrolle dabei scheinen nach den Arbeiten von Meder, Aly Bey Ibrahim und anderen die kleinen interlobulären Gallengänge zu spielen, in zweiter Linie kommt eine Art vikariierende Hypertrophie der noch erhaltenen Leberzellenbalken und endlich eine interazinöse Infiltration oder Bindegewebsneubildung in Betracht. Die Prognose ist auch bei diesen weniger rapid verlaufenden Fällen eine äußerst schlechte, wenn auch Heilungen beobachtet worden sind, die wohl auf einem Ausgang in Zirrhose (Meder, v. Kahlden u. a.) bzw. in multiple knotige Hyperplasie (Marchand, Steinhaus u. a.) beruhen. Die Therapie war in unserem Falle vor allem eine symptomatische. Im Anfange wurde Kalomel versucht, die Flüssigkeitsaufnahme wurde durch Darmeingießungen und Nährklistiere unterstützt, das Erbrechen durch Eispillen gelindert, die Unruhe durch Brom bekämpft. Nur einmal wurde bei sehr starker Unruhe, verbunden mit lautem Schreien Morphium subkutan angewendet.

Eine Uebersicht über die einschlägige Literatur findet sich unter anderem am Schlusse der $Q u$ in $c$ k e schen Ausführungen über akute gelbe Leberatrophie in Nothnagels Handbuch und in der Arbeit von $R$ e i c h m a n $n$ in der Münchener medizinischen Wochenschrift 1908, No. 18, sodaB eine besondere Anführung an dieser Stelle sich erübrigen dürfte. 